Mots. Les langages du politique

Toponymes. Instruments et enjeux

\title{
Le langage de Mai 68 dans la mémoire du cinéma de fiction. Milou en Mai, de Louis Malle
}

François de la Bretèque

\section{OpenEdition}

\section{Journals}

Édition électronique

URL : https://journals.openedition.org/mots/13802

DOI : $10.4000 /$ mots. 13802

ISSN : 1960-6001

Éditeur

ENS Éditions

\section{Édition imprimée}

Date de publication : 30 mars 2008

Pagination : 85-98

ISBN : 978-2-84788-129-5

ISSN : 0243-6450

Référence électronique

François de la Bretèque, «Le langage de Mai 68 dans la mémoire du cinéma de fiction. Milou en Mai, de Louis Malle », Mots. Les langages du politique [En ligne], 86 | 2008, mis en ligne le 30 mars 2010, consulté le 22 avril 2022. URL : http://journals.openedition.org/mots/13802 ; DOI : https://doi.org/ $10.4000 /$ mots. 13802

\section{(C) ENS Éditions}




\title{
Le langage de Mai 68 dans la mémoire du cinéma de fiction. Milou en Mai, de Louis Malle
}

\author{
Un événement n'est pas ce qu'on peut voir ou \\ savoir de lui, mais ce qu'il devient (et d'abord \\ pournous). \\ Michel de Certeau
}

Dans les derniers jours de Mai 68, Jean-Claude Carrière, de retour du Festival de Cannes, sillonne Paris en voiture en compagnie de Louis Malle. Ce dernier ne filme pas (il deviendra bientôt un des grands documentaristes français). Tous deux se contentent d'observer et d'écouter. Plus de vingt ans après (1989), Malle tourne Milou en Mai sur un scénario de Jean-Claude Carrière. Le film se passe en mai 1968 dans la campagne du Sud-Ouest ${ }^{1}$, et l'originalité du choix scénaristique est justement d'avoir pris pour cadre de l'action non les amphis de la Sorbonne ou le théâtre de l'Odéon, lieux où la parole libérée se déchainait, mais un coin reculé de la campagne française. Carrière et Malle ont choisi de présenter une perception oblique de cette période. Ils ont opté pour la province, imaginant celle-ci comme tout à fait à l'écart des évènements, ce qui n'est que très partiellement vrai².

Le film sort dans les salles en janvier 1990. Treize ans plus tard, JeanClaude Carrière rédige ses souvenirs dans Les années d'utopie (Carrière, 2003). Dans cet article, nous montrerons que ces deux « textes » sont révélateurs de la représentation du langage politique de Mai 68 tel que la postérité l'a retenu, fantasmé et transformé au gré de l'évolution sociale, politique et mentale. Carrière et Malle proposent une version psychologique et civilisationnelle de la crise de 68 en portant sur elle un regard ironique et distancié. Ce faisant, sa dimension spécifiquement politique est évacuée. Disparait également son interprétation eschatologique, dont on discute encore.

Le dialogue de cinéma, selon Michel Marie et Francis Vanoye, est avant tout le résultat du processus de production dans sa phase scénaristique,

1. On trouvera en annexe le synopsis du film, ainsi que son générique.

2. Voir le chapitre intitulé «La province », dans Dreyfus-Armand et Gervereau, 1988, p. 200-214.

Université Paul Valéry Montpellier 3 (RIRRA 21), françois.amy-de-la-breteque@univ-montp3.fr 
quoiqu'une plus ou moins grande liberté d'interprétation soit aussi laissée aux acteurs. Parmi les quatre types qu'ils distinguent à ce propos, le dialogue que Jean-Claude Carrière et Louis Malle ont écrit pour Milou en Mai entre dans la tradition la plus courante, celle de Prévert, Audiard, Aurenche ou Jeanson: un dialogue écrit qui imite le naturel de la conversation ordinaire. Milou est un film de scénariste et de dialoguiste: on y parle beaucoup, peut-être en souvenir de la fameuse "prise de parole » mise en évidence par Michel de Certeau (1994). C'est une œuvre de fiction construite pour décrire un passé proche et encore sensible. En même temps, c'est un film qu'on peut juger paradoxal: le cinéma de dialoguistes est un cinéma fabriqué, aux antipodes de la création militante et dite «spontanée» qui s'est brièvement épanouie à l'époque; il représente le cinéma institutionnel contre lequel s'étaient dressés les perturbateurs du Festival de Cannes de 1968, parmi lesquels Malle et Carrière euxmêmes (Carrière, 2003, p. 52-56).

Il arrive souvent que l'on reconnaisse la patte de l'écrivain dans ce type de dialogues : l'unité du style et des effets, sans parler du lexique, donne l'impression que tous les personnages s'expriment dans la même langue, quelque effort que le dialoguiste ait fait pour les différencier sur ce plan. Michel Marie et Francis Vanoye expriment cette idée de façon plus théorique en disant que dans un dialogue filmique, il est difficile de situer l'instance d'énonciation: elle est soit (ou à la fois) l'auteur des dialogues, soit le personnage, soit même l'acteur. Ces différentes instances peuvent faire écran entre le spectateur et la créature de fiction. Une autre spécificité du dialogue de film réside dans la place faite aux éléments infra- ou extra- linguistiques de la communication: accents, timbres de voix, gestes et environnements. Dans un film, ce que l'on voit à l'écran ne s'y trouve généralement pas par hasard; et si cela se produit, une fois le plan retenu au montage, ces éléments aléatoires entrent dans le processus de signification, fût-ce malgré le réalisateur. Dans l'article qui suit, on proposera d'abord une étude du vocabulaire avant d'en venir aux formes de communication non verbales mises en scène à l'écran.

\section{Le vocabulaire dans Milou en Mai}

\section{Le texte des bulletins radiophoniques}

On sait que la radio, et notamment le poste à transistors, fut le medium privilégié des évènements de Mai 68 . On se souvient du rôle pilote d'Europe ${ }^{\circ}{ }^{\circ} 1$, adoptée par les Français à une époque où les chaînes du monopole public, contrôlées par le gouvernement, avaient perdu la confiance des auditeurs. Le film de Louis Malle rend bien compte de ce fait, mais en livre une version transformée. Voici trois des six bulletins que l'on y entend : 


\section{Premier bulletin (entendu par Mme Vieuzac, 49 secondes) ${ }^{3}$ :}

Très violente manifestation hier soir au Quartier Latin. Une vingtaine de voitures brûlées. Des barricades érigées. Des affrontements qui ont fait douze blessés parmi les forces de l'ordre. Une cinquantaine d'arrestations. L'Union nationale des étudiants de France dénonce ce matin les brutalités policières. Dans le même temps, le préfet de police affirme que les étudiants sont maintenant encadrés par des milices armées. Manifestations aussi en province où, pour la première fois, des paysans ont érigé des barricades autour de [Nantes], engageant ainsi le monde paysan derrière les étudiants et les ouvriers ${ }^{4}$.

\section{Deuxième bulletin (écouté par son fils Georges, 23 secondes 58) :}

La grève s'étend maintenant à tout le pays. Déjà dix millions de travailleurs en grève. Dans tous les magasins des stocks ont été pris d'assaut, prioritairement le sucre et la farine. à Paris, le prix de la pomme de terre nouvelle est passé en 48 heures de 0,90 à 3 francs le kilo. Dans les pharmacies également, l'approvisionnement est difficile. à la Banque de France, la diminution des réserves a entrainé... [inaudible] Au plan politique, tout le monde attend maintenant la déclaration du général de Gaulle. Dans ce contexte, François Mitterrand s'est déclaré prêt à assumer ses responsabilités; Daniel Cohn-Bendit, qui avait momentanément quitté la France, s'est vu refuser l'entrée du territoire. Il est désormais interdit de séjour [suite inaudible $]^{5}$.

\section{Cinquième bulletin (écouté par Georges, 62 secondes) :}

Ce matin, les locaux de la fédération française de football ont été occupés à leur tour par des manifestants. Fernand Choiseul, les échos de cette manifestation?... - Vous m'entendez, Jacques Paoli? [On entend scander dans le fond :] « Le football aux footballeurs!»

Les bulletins incorporés à Milou ne sont pas authentiques: ils ont été rédigés pour le film (comme Agnès Varda l'avait fait dans Cléo de cinq à sept), ce qui explique leur côté synthétique et leur intemporalité sans doute intentionnelle. Les auteurs, sans chercher à retrouver le langage radiophonique de l'époque,

3. Le texte de ce bulletin n'est pas conforme à celui du scénario, qui poursuivait ainsi : "La grève s'étend à tout le pays. SNCF, RATP, PTT, tous les services publics cessent peu à peu le travail. Plusieurs millions de travailleurs sont en grève. On ne peut plus se déplacer autrement en France qu'en voiture particulière, et l'essence commence à manquer en province, les pompistes sont pris d'assaut. Les Parisiens font la queue dans les magasins d'alimentation et stockent des denrées de base comme le café et le sucre.» (Op. cit., p. 10). Le réalisateur, jugeant ces informations didactiques et redondantes, a préféré les signifier par des situations que vivent les personnages.

4. Il s'agit probablement de la manifestation de la nuit du 9 au 10 mai. Voir chronologie dans Dreyfus-Armand et Gervereau, 1988, p. 284-303.

5. Ce second bulletin est composite. Il ne figure d'ailleurs pas dans le scénario. Le nombre de grévistes y correspond aux évaluations à partir du 20 mai. Cohn-Bendit est reconduit à la frontière le 24 mai, jour du premier discours du général de Gaulle. François Mitterrand se déclare candidat le 28 mai. On mesure à ces détails le travail de synthèse auquel se sont livrés les deux auteurs. 
se contentent d'un échantillon minimal : barricades, manifestants, manifestation, grève, soit un lexique peu marqué et qui reste très général. On peut tout au plus relever, comme marqueurs de la période, forces de l'ordre (euphémisme alors nouveau qui se lexicalisera rapidement), et la série figée étudiants, ouvriers, paysans, reflet d'une volonté unitaire qui fut l'un des grands fantasmes de Mai 68.

\section{Le vocabulaire des personnages}

Le scénario emprunte évidemment son vocabulaire à des sources multiples et s'efforce de doter chacun des énonciateurs d'un lexique particulier permettant de l'individualiser. Cependant, il ne faut pas négliger le travail de sélection et de réfraction auquel il se livre, les scénaristes élaborant ainsi, fût-ce à leur corps défendant, "leur» Mai 68. Comme on ne saurait dire ce qui revient à Malle et ce qui revient à Carrière (de l'aveu même de ce dernier), on traitera ceux-ci comme un énonciateur collectif.

Il existe un socle commun au discours des différents personnages. Un examen du vocabulaire qu'ils partagent, aussi réduit soit-il, nous livre, sous la forme d'un champ lexical que la mémoire a figé et embaumé, cette vision de Mai 68 élaborée vingt ans plus tard. Le vocabulaire des personnages est aussi, partiellement, celui qui est prêté aux journalistes de la radio : les auteurs n’ont pas cherché à accentuer les différences. Il existe néanmoins des termes qui ne figurent que dans les dialogues: Pierre-Alain parle une fois des CRS et des grenades lacrymogènes, une autre fois des matraques. Révolution (également absent des bulletins radiophoniques) est employé deux fois par Camille, notamment quand elle avance que «c'est la révolution qui a tué » sa grand mère, une fois par le curé qui déclare, à propos des lycéens du coin : «Ils n’ont pas le temps [de venir chercher des tomates], ils font la révolution », à deux reprises parFrançoise ("Vive la révolution »), et de façon plus neutre par Georges qui fait alors référence à 1789. Camarade n'apparait qu'une fois (incidemment, lorsque Camille et Paul, traversant en voiture une manifestation, se font interpellerainsi par un jeune ouvrier qui leur remet un tract), de même que capitalistes (dans un énoncé de Camille). D'autres termes sont absents: contestation, subversion, enragés, chienlit, gauchistes, staliniens, réacs, fachos, répression...

Les acteurs et les sympathisants du mouvement ne sont représentés dans Milou que par Pierre-Alain, le fils de Georges, engagé dans le mouvement étudiant à Paris, et incidemment par le curé. Le vocabulaire de Pierre-Alain est peu marqué sur le plan politique. Une seule tirade, au premier dîner où il s'oppose à son père, prend une petite coloration militante: "C'est normal qu'on s'organise! Et c'est normal que la bourgeoisie résiste!» Et d'ajouter: «Il faut toujours faire le bonheur des gens malgré eux, c'est bien connu», phrase qui le classe parmi les idéologues radicaux et donc dangereux. Mais les termes 
qui reviennent le plus souvent dans sa bouche (désir, joie, fête, spontané) appartiennent à un autre champ sémantique. Hormis spontané, chargé en 1968 d'une valeur spécifique, ce sont des expressions du langage courant qui, avec le temps, ont pris une connotation particulière: elles renvoient de manière large aux années soixante, au flower power comme l'indique Carrière dans son récit'

La rhétorique d'Alain est remarquable par son recours aux formulations négatives: «C'est ça qui est bien justement! Le fait qu'ils ne réclament rien! Rien de précis! « «Q'est-ce qu’ils [les étudiants] disent? C'est très simple. Ils disent: on en a assez du fric, du profit, assez du pouvoir des pays riches, assez de la consommation, assez d'épuiser la terre, et ils disent: arrêtons-nous ... ». On ne sait pas ce qu'on veut, ce qui énerve les adultes, mais on sait au moins ce qu'on ne veut pas. Michel de Certeau avait observé dès 1968 cette forme d'expression et rappelé que « toute négation se contente d'inverser les termes de l'affirmation qu'elle construit». Cette récurrence des formules négatives traduisait, selon l'historien, la disparité entre « une expérience fondamentale et le déficit de son langage, entre la "positivité" d'un vécu et la "négativité" d'une expression qui, sous la forme du refus, semble le symptôme plutôt que |'élaboration de la réalité qu'elle désigne »' ${ }^{7}$. De son côté, Carrière écrira dix ans plus tard: "L'utopie française de Mai 68 avait ceci de particulier qu'elle détruisait sans construire. $»^{8}$ Et d'ajouter que c'est cela qui la rendit la plus féconde et la plus réaliste de toutes.

Les adversaires du mouvement sont représentés par les Boutelleau, patrons de l'usine (qui n'apparaissent que dans une séquence de la dernière partie) et de façon plus substantielle par Camille et par Paul. L'une est une mère de famille catholique et coincée, l'autre appartient à la catégorie des lâches et des fuyards: il est absent pendant la plus grande partie du film. S'y ajoute le camionneur Grimaldi, qui a amené Pierre-Alain dans son véhicule tout en ne partageant aucune de ses idées. Soit une représentation volontairement équilibrée : deux groupes de bourgeois et un prolétaire. Ni Camille ni Grimaldi ne sont antipathiques, à l'inverse des Boutelleau, véritables caricatures à la Prévert (ils font beaucoup penser au personnage de Jules Berry dans le film de Renoir, Le Crime de Monsieur Lange).

6. Les années d'utopie est sous-titré: Des fleurs, des pavés et des tanks. Il est question du «flower power» sur les campus américains p. 38 et suivantes. Jean-Claude Carrière a intitulé un des chapitres "L'illusion du plaisir»: "Ce fut la grande illusion de Mai. Que la révolution fût l'ennemi assuré du plaisir, que l'hédonisme fût à l'opposé du communisme, nos idéologues avaient choisi de l'écarter de leur jeune pensée. [...] Ils ne pouvaient aller de l'avant qu'en répétant comme une incantation les mots "désir" et "révolution" jusqu'à en faire, une fois de plus, des objets mythiques.» (Ibid., p. 78). En écrivant cela, Jean-Claude Carrière fabrique luimême, sans s'en rendre compte, une mythologie.

7. Michel de Certeau, 1994, p. 45. Texte original: Prendre la parole, Paris, Desclée de Brouwer, 1968.

8. Carrière, 2003, p. 73. 
Or, abstraction faite des niveaux de langue, le vocabulaire de Camille et celui de Grimaldi se ressemblent étrangement. Camille (Miou-Miou) affecte un langage décontracté, mais on sent l'élocution et le phrasé d'une grande bourgeoise 9 . Grimaldi - interprété par un ancien des «Nuls» de Canal $+{ }^{10}$ est un prolétaire qui s'amuse à choquer le bourgeois, mais un prolétaire de cinéma qui s'inscrit dans la tradition des films populistes français. Il parle très peu argot, sa syntaxe est à peine relâchée. De fait, il existe une convergence entre le langage de ces personnages situés aux deux extrêmes de la palette sociale du film. Camille parle des «petits cons de la Sorbonne», Grimaldi des "petits connards». Paul voit en eux des «enfants gâtés »" ${ }^{11}$. Camille pense que les grévistes «font les malins», qu’il leur manque «des taloches», " un peu de poigne», et voudrait faire intervenir l'armée. Grimaldi juge que «Paris, c'est le bordel ». La différence, c'est qu'il semble s'en accommoder et même y trouver des avantages, obsédé qu'il est par les rencontres sexuelles (dont il parle bien plus qu'il ne les concrétise).

Du côté des neutres et des sceptiques, on trouve Milou et Georges, les deux frères. Ils occupent des positions symétriques et inverses: Georges est un intellectuel surmené, secrètement dépressif, guindé et institutionnel; Milou est sanguin, charnel, épicurien et ironique.

Le vocabulaire de Milou est peu marqué. C'est lui qui objecte au curé: «On n’a pas beaucoup vu Dieu sur les barricades », à quoi celui-ci répond: "Parce qu'on ne sait pas regarder», faisant ainsi référence à la version pour ainsi dire religieuse des évènements de Mai 68, celle de Maurice Clavel comme de Gabriel Matzneff. Milou n'est aucunement mystique, et c'est la seule fois où il est question de Dieu et de spiritualité dans ce film très laïque. Mais le scepticisme de ce personnage est équilibré par sa réponse à Paul: «Ils [les étudiants] tiennent le coup, quand même!», qui traduit une certaine admiration. C'est la sympathie qui l'emporte finalement, une sympathie à distance, désengagée. Dans une scène non retenue, le scénario prévoyait que Milou demande à Pierre-Alain de lui «raconter Paris» (Malle et Carrière, 1990, p. 86). En fin de compte, il n'exprime directement ses opinions qu'une fois, à la fin du film, après que Boutelleau a pollué la rivière en vidangeant les cuves de son usine. Milou lui lance à cette occasion une bordée d'injures: «Assassin! Pollueur! Fumier! Dégueulasse! Ordure! Maquereau! Exploiteur! Industriel! Hypocrite!

9. Écouter par exemple le ton inénarrable de sa première réplique: «Vous voulez des petitsbeurre, les enfants?»

10. À côté d'acteurs confirmés du cinéma français comme Piccoli et Duchaussoy, le film intègre, dans leurs premiers rôles pour certains, des acteurs venus du café-théâtre, ce qui informe sur les registres linguistiques exploités. Miou-Miou est une ancienne du Café de la Gare et Valérie Lemerciervient du Splendid. La plus ancienne génération est représentée par Paulette Dubost, actrice de Renoir, en hommage à celui-ci dont s'inspire à l'évidence le ton du film.

11. Peut-être en écho à l'analyse que Georges Pompidou développe dans Le nœud gordien, Paris, Plon, 1974. 
Mercanti ! Invectives banales et non spécialisées dans le domaine politique, à l'exception peut-être d'exploiteur et - plus inattendu en fonction d'insulte d'industriel. Cette série s'apparente plutôt aux tirades du capitaine Haddock qu'à celles des manifestants gauchistes. Mais on peut relever la connotation écologique avant la lettre de plusieurs de ces expressions ("pollueur», «fumier», «industriel»), ainsi que de la situation représentée (la dégradation d'une paisible rivière à écrevisses), alors qu'on aurait pu s'attendre à ce que le scénario mette ici en exergue l'exploitation de la classe ouvrière par un petit patron. C'est un des détails par lesquels le film marque sa date.

Georges est le personnage dont le vocabulaire est le plus riche, le plus caractéristique et, partant, le plus intéressant à étudier. II maîtrise, de par sa profession, le langage de l'analyse politique et l'étale non sans suffisance. Ses premières tirades, lors du dîner, en témoignent. On y entend les expressions révolution, mouvement ouvrier, la gauche, gouvernement, engagement, mouvement («le mouvement s'élargit»)..., bref, beaucoup de termes abstraits. Il emploie aussi le terme chaos pour évoquer ce vers quoi risque d'évoluer la situation politique et sociale, utilisant ainsi un mot assez gaullien, mais évitant chienlit, qui serait plus spécifiquement associé à Mai 68 . Selon le scénario ("Les enfants mangent et écoutent comme au spectacle», Malle et Carrière, 1990, p. 40), son discours est un quasi-monologue, et seule Camille trouvera une objection: "Qu'ils fassent intervenir l'armée!» Mais Georges est l'incarnation d'une position balancée et prudente: il craint les excès et les débordements du mouvement social tout comme il déplore l'impuissance et l'aveuglement du pouvoir gaulliste. Plus loin dans le film, ayant écouté l'allocution du 24 mai, il juge de Gaulle «mauvais»: "Il nous refait le coup du référendum », estime-t-il en faisant la moue; le discours est «loupé». Après la fuite à Baden-Baden, il porte un jugement plus sévère: "Qu'est-ce qui restera de de Gaulle, au fond? Pas grand-chose [...] il n'a rien vu venir, absolument rien. ${ }^{12}$ Il annonce qu'il est en train d'écrire un livre dont le sujet est justement l'aveuglement du gaullisme. Son titre: La Cassure; son thème : «On n'a rien prévu pour la jeunesse». Georges est fier de son pronostic: «Ce qui se passe confirme exactement ce que je dis dans mon bouquin. "Sa version représente assez bien celle des gaullistes orthodoxes, même s'il n'appartient sans doute pas à cette mouvance . Pour autant, Georges n'a pas plus confiance dans l'issue du mouvement en cours. Une deuxième occasion lui est donnée d'exposer ses idées quand il s'oppose à son fils lors d'un repas à la cuisine où l'on mange les écrevisses pêchées par Milou. Il essaie de dissocier les étudiants des ouvriers, les uns ayant des revendications concrètes, les autres pas: il met ainsi le doigt sur une des grandes inquiétudes des leaders

12. On pense par exemple à l'article d'Hubert Beuve-Méry dans Le Monde du 25 mai : voir dans Dreyfus-Armand et Gervereau, 1988, p. 247. 
historiques de Mai. Il ne croit pas à la spontanéité du mouvement et évoque les "groupuscules», parle de "comités», de «maoïstes» et de "situationnistes» : c'est le seul moment du film où l'on entend parler de ces réalités politiques de Mai 68.

Cette posture médiane d'un observateur perplexe traduit celle du journal Le Monde au moment des évènements ou, plus exactement, le souvenir que l'on en a conservé: Georges fait penser à Pierre Viansson-Ponté et à son fameux éditorial du 2 mai ("La France s'ennuie»). D’un autre côté, il se présente comme une assez exacte synthèse des hésitations des journalistes du Monde (Nicole Bernheim, Jacques Nobécourt, Frédéric Gaussen, Guy Herzlich) face à un mouvement dont les références sont inconnues, et rappelle leur tentative de le ramener à du connu, de l'intellectualiser, comme l'a bien montré Marie-France Raflin . Evacuant à la fois les revendications proprement politiques et les références alors méconnues de lui (tiers-mondisme, situationnisme, autogestion notamment), il en vient à considérer le mouvement comme "une contestation dont les visées sont très générales» (ibid.). Cette perplexité rejoint celle de Jean-Claude Carrière : ce journaliste en passe d'être limogé est aussi, a-t-il déclaré, son personnage préféré.

\section{La désignation des évènements}

On a vu que les évènements de Mai 68 ne sont une «révolution» que pour trois des personnages, dont une enfant (Françoise) et une adversaire farouche (Camille) dans la bouche de qui ce désignant est ironique. Georges, en raison de sa culture historique (il se réfère à la France de 1788), envisage que le mouvement finisse par prendre cette forme, mais n'y croit pas trop au fond. Seul le curé, personnage secondaire mais révélateur, emploie ce mot avec conviction $^{13}$. Les adversaires, eux, parlent de bordel ou de chaos.

Le seul terme sur lequel tous s'accordent, et qui est proféré par tous les personnages, est plaisir. C'est Pierre-Alain qui l'introduit, mais il circule et devient le dénominateur commun au moment de la fête. Il s'en faut de peu que celle-ci ne vire à la soirée échangiste : le terme, en effet, signifie avant tout plaisir sexuel14. Georges lui-même le reprend: "Le plaisir! Le plaisir pur! Sans arrière-pensée! Donc le bonheur!» Terme fédérateur qui réussit presque à produire les effets qu'il décrit : rassembler tous les personnages dans un communautarisme hédoniste en effaçant les différences de condition.

13. À l'inverse, en 2003, Carrière écrit: «Révolution. Le mot courait partout. Il surgissait dans chaque paragraphe. Nous étions tous des révolutionnaires [...].» (p. 64) La chronique écrite révèle le souvenir d'un autre langage que celui du dialogue du film. Mais peut-être faut-il imputer cet écart au fait que le film est situé dans un coin de province alors que la chronique est parisienne.

14. Même restriction du sens dans le texte de Carrière, p. 76 et suivantes. 
Ce que le film retient est donc la conception de Mai 68 en tant que grand happening qui aurait touché toutes les couches sociales, mais brièvement. Car au bout du compte, la mise en commun ne se fera pas; ni celle des couples et des corps, ni celle de l'héritage de Mme Vieuzac. L'épisode échangiste, ébauché, sera interrompu par l'arrivée des Boutelleau; et la vie soi-disant naturelle dans les bois prendra fin avec le retour de l'essence, pendant le week-end de la Pentecôte.

\section{Autres composantes de l'expression filmique}

Au-delà des énoncés qui font référence à la situation historique, d'autres éléments renvoient à la mémoire des évènements. Ils se situent dans le dialogue, mais aussi dans les images et les sons. L'ensemble de ces indices paraverbaux et situationnels (images, paroles, bruits, gestes, cadres, évènements, etc.) compose une image diffuse de la mythologie de Mai 68. "L'intelligibilité d'un film passe à travers trois instances principales, écrivent Michel Marie et Marc Vernet, l'analogie perceptive, les “codes de nomination iconiques", ceux qui servent à nommer les objets et les sons, enfin, les figures signifiantes proprement spécialisées qui constituent [ce que l'on appelle communément] le langage cinématographique» (Aumont et al., 1983, p. 131). Le second et le troisième niveaux concernent notre propos dans la mesure où des images, des sons, des effets de montage peuvent être décodés au niveau verbal et renvoyer ainsi au vocabulaire politique de l'époque.

\section{Bruits et rumeurs}

Certaines représentations sont construites par les propos des personnages. Des rumeurs circulent, à propos notamment de la brutale pénurie des biens de consommation: par exemple, l'essence manque et le fossoyeur, qui en avertit Milou, lui rapporte que l'épicier du village « en a rempli sa baignoire». D'autres rumeurs qui ont circulé dans ces semaines-là, rappelées par JeanClaude Carrière dans Les années d'utopie (2003, p. 80), ont posé les bases d'une légende noire de Mai 68. Certaines d'entre elles, parvenues jusqu'au fond de la province, sont livrées dans le film: Claire, la nièce de Milou, a entendu dire qu'il y a eu des victimes «mais qu'ils cachent les corps ». L'obsession du premier mort possible est très présente ${ }^{15}$ : Camille puis Georges l'expriment. Il circule aussi quantité de bruits sur les orgies sexuelles qui se dérouleraient dans les couloirs de la Sorbonne et à l'Odéon, ce qui excite beaucoup le camionneur Grimaldi. En revanche, rien n'est retenu de ce qui fit le Livre

15. Elle fut effective, comme le montrent les mémoires de Maurice Grimaud (1977). 
noir de 68 , ni la répression policière, ni certains détails comme les fameux «Katangais» de la Sorbonne que Jean-Claude Carrière cite en revanche dans son livre (2003, p. 81), mais avec une erreur sur le sens de cette expression ${ }^{16}$.

\section{Sons et musique}

Les échos lointains des manifestations parisiennes parviennent jusqu'à ce tranquille village du Sud-Ouest, où l'on entend parler de matraques, de pavés, de CRS. Mais dans la bande-son, les allusions peuvent se faire encore plus directes. L'Internationale, incontournable référence, intervient à plusieurs niveaux. On l'entend dans les deux manifestations que montre le film, chantée par les ouvriers de Boutelleau et les élèves du lycée Gambetta (rebaptisé «Lycée Che Guevarra » sur la suggestion du curé); on l'entend aussi en fond sonore des reportages radiophoniques (nous dirons qu'elle est ici directement diégétique). Elle est aussi reprise comme par inadvertance par l'un ou l'autre des personnages (Milou, Georges), à la façon d'une rengaine entêtante (elle est alors indirectement diégétique). Enfin, on l'entend sous une forme arrangée dans l'accompagnement sonore de la scène de grande euphorie collective (elle est alors extradiégétique et remplit une fonction de commentaire). L'hymne de l'Internationale ouvrière décolle ici de ses références historiques et politiques et devient une mélopée du temps. En revanche, ce film ne fait aucune référence à la musique rock, pas même aux chansons des Beatles, chose peu vraisemblable y compris en province, d'autant plus que deux des personnages sont jeunes et viennent de Paris (Marie-Laure et Pierre-Alain) ${ }^{17}$. La musique du film, assez discrète, est de Stéphane Grapelli, un des piliers du jazz français, mais peut être sentie comme historiquement désuète en cette fin des années soixante.

\section{Images et objets emblématiques}

Il en va de même de deux emblèmes visuels de Mai 68: les drapeaux rouges et les banderoles. On voit les premiers dans les deux manifestations déjà mentionnées, ainsi qu'un drapeau noir pendant la manifestation lycéenne. Après celle-ci, «les deux jumeaux ramassent un drapeau rouge oublié par les lycéens et le font claquer au vent», geste qu'on dirait repris du Charlot des Temps Modernes. Quant aux banderoles et aux slogans ils restent discrets: "Usine en grève », "de Gaulle à Colombey», "Dix ans, ça suffit», "Nous sommes tous des juifs allemands». Le premier slogan, banal, rappelle aussi les usines

16. Les «Katangais » de la Sorbonne n'avaient rien à voir avec un quelconque tiers-mondisme, pas plus qu'il n'étaient, comme le voulait la légende, des mercenaires passés au service de la subversion. Voir Pagès, 1998, p. 108.

17. Et alors qu'on écoute Europe $n^{\circ} 1$, la station de «Salut les copains». 
occupées de l'été 1936. Les deux suivants sont conjoncturels: leur sens s'est vidé, ils ne parlent plus guère au spectateur de 1990. En revanche, le dernier a acquis un statut de modèle, il s'est universalisé au fil du temps.

À ce propos, on doit relever que le seul leader du mouvement dont le nom soit mentionné dans le film est Daniel Cohn-Bendit ${ }^{18}$. Il est évoqué trois fois. On vient d'indiquer la première. Le bulletin radio de la $62^{\mathrm{e}}$ minute annonce son interdiction d'entrée sur le territoire. Plus loin, au pire de la crise, les Boutelleau, qui pensent que de Gaulle "est peut-être mort», annoncent que "l'anarchiste allemand est rentré! Oui, Cohn-Bendit! Il a donné une conférence de presse à la Sorbonne!» (événement survenu le 25 mai). L'expression, présente sous ses deux variantes (juif allemand, anarchiste allemand), a marqué les mémoires. Selon Marie-France Raflin, c'est le travail à chaud des journalistes du Monde qui a donné cette coloration épique aux évènements, en faisant ressortir les principales figures de la révolte.

Deux autres emblèmes de 68 sont mis en évidence. D'une part, le «joint » qui circule entre les personnages, apporté par l'Anglaise Lily; tous y goûtent sauf le camionneur Grimaldi qui préfère sa Gauloise (« Moi, je fume français »); le «swinging London » est proche des esprits. D'autre part la «pilule», dont on parle beaucoup mais qu'on ne voit pas. Autorisée par la loi Neuwirth de 1967 (non encore entrée en application à l'époque), elle est le vecteur et le symbole de la libération sexuelle. En réponse à la petite Françoise qui lui demande ce que c'est, Milou répond: "C'est le progrès. » (Malle, Carrière, 1990, p. 67) Les questions que pose cette enfant de façon récurrente obligent les adultes à passer par le langage et à se confronter au vrai sens des choses, des conduites et des slogans: «C'est quoi le smig? Tu l'aimes, toi, de Gaulle? Pourquoi elle a pas d'enfant, tante Claire? Papy, c'est quoi le foutre? » ...

\section{Contrepoints}

Milou en Mai, film de scénaristes, est remarquable par la forte présence de ces énonciateurs derrière les propos des personnages. Toute l'histoire est tenue à distance, une distance qui est la marque de l'ironie. Une technique revient systématiquement, celle du contrepoint. On l'observe dans la succession des répliques, et c'est alors un vieux procédé auquel ont recouru les scénaristes français, les Jeanson, les Aurenche et les Bost que Truffaut avait si vivement critiqués. On l'observe aussi dans la confrontation des paroles et des images.

Exemples du premier cas: (Georges): "La gauche essaie de prendre le train en marche... / Tu me passes un petit peu d'os à moëlle?»; (Claire) : "Il paraît qu'il y a des morts, mais qu'il cachent les corps, la nuit!.. / Tu me passes

18. Du côté des hommes politiques, outre de Gaulle et Pompidou, seul Mitterrand est cité, ce qui n'est pas indifférent en 1988, évidemment. 
le gros sel?»; (Georges, parlant de son livre La Cassure) : «On n’a rien prévu pour la jeunesse! Rien!.. / C'est quand l'enterrement, au fait?» (Il s'agit de celui de Mme Vieuzac, mais aussi, implicitement, de celui de de Gaulle).

Exemples du second cas: au moment où Camille déclare que Pierre-Alain est occupé à "lancer des pavés sur les agents», les jumeaux envoient une boulette de pain en direction de Marie-Laure. Les coupures d'électricité symbolisent une France "plongée dans le noir», comme le remarque Boutelleau, mais c'est une obscurité chaude et complice pour les protagonistes et le retour de l'électricité signifie la fin de la parenthèse. Au début du film, Milou essaie d'endormir les abeilles de ses ruches en leur lisant en latin un passage des Géorgiques. Il est difficile de ne pas penser aux mandarins de la Sorbonne qui vont se faire contester: "On entend l'écho lointain d'un orage. Les abeilles se mettent à voler nerveusement.» (Malle, 1990, p. 10) Bon exemple de métaphore réalisée par l'image et comparable au fameux "Il pleut bergère » de 1789.

Enfin et surtout, l'héritage de Mme Vieuzac et le partage qui se négocie avec âpreté entre les enfants prennent, à l'échelle de tout le film, une valeur métaphorique. La disparition de la vieille dame peut figurer celle de la vieille France rurale : Malle et Carrière ne peuvent s'empêcher de faire sentir qu'ils en éprouvent une certaine nostalgie. Mais ils se résignent, comme leurs personnages: on ne peut revenir en arrière. Qu'est-ce qui va remplacer cette structure familiale et sociale figée? Un espoir se fait jour, celui d'une vie collective généreuse et égalitaire: la propriété ne sera pas partagée, on y vivra en communauté. Au plus fort du rêve utopique, de partage il n'est plus question. Mais la dernière séquence fait comprendre que le principe de réalité a fini par l'emporter. La propriété de madame Vieuzac (si l'on décode en gros), c'est la France de de Gaulle; la phase de vie communautaire, c'est la « révolution »; et le retour aux réalités du partage, c’est la réintégration dans la société capitaliste et marchande.

C'est Louis Malle qui a eu l'idée initiale de la mort de la vieille dame et de la maison que l'on veut vendre (souvenir probable du film de Roger Leenhardt, Les Dernières vacances). Toute la suite est le résultat d'un travail à deux mains. Milou en Mai, film très écrit, est bien loin de l'improvisation que l'on associe, à tort, à la Nouvelle vague. C'est sans doute là son point faible sur le plan artistique, mais ce défaut permet d'instruire notre propos: sous les dehors de cette gentille fable provinciale, c'est bien le discours de ses auteurs, Louis Malle et Jean-Claude Carrière, qui se fait entendre, écho de ce qui, dans un certain cercle d'artistes et d'intellectuels français (et même au-delà), est devenu vingt ans après les évènements le mythe de Mai 68.

Cette version cinématographique de Mai 68 concorde avec celle du livre Les années d'utopie, et met en exergue des préoccupations qui seront davantage celles des années soixante-dix, même si l'on peut montrer qu'elles étaient 
en germe dans les slogans de Mai : "Sexand drugs et autres ras-le-bol envers l'ordre établi, familial, urbanistique, productiviste, consumériste, etc. ». Kristin Ross a procédé à une dénonciation sévère de cette interprétation qui tend à s'imposer aux esprits d'aujourd'hui, qu'ils se définissent comme sympathisants ou comme adversaires de l'esprit soixante-huitard. Refusant que l'on attribue au mouvement une dimension purement culturelle ou spirituelle, elle retourne au langage préservé dans les tracts et les films militants de l'époque. Selon elle, c'est avant tout d'un mouvement de contestation politique qu'il s'agit. écrivant peu après les grandes grèves de décembre 1995, elle pronostique un retour de ces mots d'ordre dans le mouvement social et, ce faisant, donne d'ailleurs une autre version, antithétique, de la mémoire de Mai 68.

Mais on voit bien que tel n'était pas, en 1988, le souci de Louis Malle et de Jean-Claude Carrière. Aujourd'hui, cette version rose l'emporte toujours au cinéma, comme on le voit dans le film de Bernardo Bertolucci, Innocents (2003), à moins que l'on en propose une contrepartie noire, celle de Philippe Garrel dans Les Amants réguliers (2005).

\section{Références}

Aumont Jacques, Bergala Alain, Marie Michel, Vernet Marc, 1983, Esthétique du film, Paris, Nathan Université.

BACOT Paul, 1986, «“Crise salutaire” ou “divertissement triste” ?, À la recherche d'une certaine idée de Mai 68 », Pouvoirs, n 39, Mai 68, p. 59-70.

CARRIÈre Jean-Claude, 2003, Les années d'utopie, 1968-1969. New York-Paris-PragueNew York, Paris, Plon.

De CERTEAu Michel, 1994, La prise de parole et autres écrits politiques, édition établie et présentée par L. Giard, Le Seuil (Points).

GrIMAUd Maurice, 1977, En mai, fais ce qu'il te plaît, Paris, Stock.

Malle Louis, CARrière Jean-Claude, 1990, Milou en Mai. Scénario, Paris, Gallimard (NRF).

Marie Michel, Vanoye Francis, 1983, "Comment parler la bouche pleine? Note méthodologique pour l'étude du dialogue des films narratifs», J.-P. Simon, M. Vernet éd., Communications, $\mathrm{n}^{\circ} 38$, énonciation et cinéma.

Ory Pascal, 1983, L'Entre-deux-Mai. Histoire culturelle de la France, Mai 1968 - Mai 1981, Paris, Le Seuil.

PAG Ės Yves éd., 1998, No copyright, Sorbonne 68, graffiti, Genève, Verticales.

RAFLIN Marie-France, 1988, "Les manifestations de la révolte en mai 1968: résurgence des extrêmes gauches marginalisées ou émergence d'une culture antiautoritaire?", Révolte et société, F. Gambrelle, M. Trebitsch éd., Paris, Histoire au présent, p. 270-278. 
Ross Kristin, 2005, Mai 68 et ses vies ultérieures, Paris, Le Monde diplomatique / Complexes.

Winock Michel, 1987, Chronique des années soixante, Paris, Le Seuil.

\section{Annexes}

\section{Générique du film Milou en Mai}

Scénario: Louis Malle et Jean-Claude Carrière. Réalisateur: Louis Malle. Images: Renato Berta.Musique:Stephane Grapelli. Interprètes: Michel Piccoli (Milou), Miou-Miou (Camille), Michel Duchaussoy (Georges), Dominique Blanc (Claire), Harriet Walter (Lily), Paulette Dubost (Mme Vieuzac), Bruno Carette (Grimaldi), Valérie Lemercier (Mme Bertheleau). Sorti à Paris le 24 janvier 1990.

Synopsis du film Milou en Mai (Louis Malle, 1990, "Prière d'insérer»)

Une vieille dame meurt, dans une propriété du Sud-Ouest. Elle y vivait seule avec son fils Milou et une jeune servante. Toute la famille accourt, comme toujours dans ce cas. à une différence près, c'est que nous sommes en mai 1968 et que les évènements de Paris, grossis, déformés par la distance, vont entrainer les uns et les autres à des réactions imprévues.

D'abord, c'est comme si le désordre autorisait chacun à se livrer à ses instincts, à son égoïsme. Et soudain, cela tourne à la fête et pour un peu, le vieux domaine deviendrait un phalanstère. Puis la peur s'installe: le pouvoir gaulliste s'effondre, la guerre civile est proche. Pris de panique, les voici qui s'enfuient, vont se cacher dans la montagne. Quand tout rentre dans l'ordre, on pourra enfin enterrer la vieille dame et abandonner Milou à sa solitude.

Un monde bourgeois bien fatigué et qu'une secousse fait trembler sur ses bases, abolissant conventions et convenances, voilà ce que peint Louis Malle. Devant la cruauté légère des personnages, on peut penser à Goldoni, à Marivaux. Le décor du Sud-Ouest, que le cinéaste connait si bien, sert merveilleusement cette comédie douce-amère. 